\title{
Theoretical Investigation of Transmission and Dispersion Properties of One Dimensional Photonic Crystal
}

\author{
Ouarda Barkat \\ Department of Electronics, University of Constantine 1, Constantine, Algeria
}

Email address:

barkatwarda@yahoo.fr

To cite this article:

Ouarda Barkat. Theoretical Investigation of Transmission and Dispersion Properties of One Dimensional Photonic Crystal. Journal of Electrical and Electronic Engineering. Vol. 3, No. 2, 2015, pp. 12-18. doi: 10.11648/j.jeee.20150302.11

\begin{abstract}
In this work, we demonstrate via numerical simulation the general design for one dimensional photonic crystal (1DPC). In the design procedure, the transfer matrix method and Bloch theorem are used to determine the transmission coefficient and dispersion relation of (1D- PC) structure for both TE (transverse electric) and TM (transverse magnetic) modes. Results obtained showing the effect of the filling factor as well as the incident angle on the photonic band gap width. The analysis is carried out using MATLAB software tool. The accuracy of the analysis is tested by comparing the computed results with measurements published data.
\end{abstract}

Keywords: Photonic Band Gap, Transfer Matrix Method, Bloch Theorem, Transmission, Dispersion

\section{Introduction}

Photonic crystals (PCs) structures have attracted increasing interest in recent years [1-5]. Various materials and techniques have been employed in order to obtain one, two, and three dimensional photonic crystals [6-9]. These structures designed to control the propagation of electromagnetic waves in the same way as the periodic potential in semiconductor crystals [10-12]. Particularly, one dimensional photonic crystals have been known for several decades as Bragg mirror. Interference of the Bragg scattering is considered as a cause of the Bragg gap or band gap. The periodicity creates the band gaps depend on some parameters, as the dielectric contrast between the employed materials, and the filling factor of the elementary cell [13].

One dimensional photonic crystal (1D- PC) structures have a number of useful properties, which are employed as low-loss optical waveguides, dielectric reflecting mirrors, optical switches, optical limiters, optical filters etc. It has been demonstrated experimentally and theoretically that one-dimensional PBG have complete omnidirectional PBGs. Various materials have been employed in order to obtain one photonic crystals (1D) as: $\mathrm{Si} / \mathrm{SiO}_{2}, \mathrm{SiO}_{2} / \mathrm{TiO}_{2}, \mathrm{Na}_{3} \mathrm{AlF}_{6} / \mathrm{ZnSe}$, $\mathrm{Na}_{3} \mathrm{AlF}_{6} / \mathrm{Ge}$ [5, 14-18]. Silicon has been the choice for microelectronics technology because of various reasons such as its cost, compatible with mass production and availability. For this reason, we are study the transmission coefficient and dispersion curves of (1D- PC) structures composed of $\mathrm{Si} / \mathrm{SiO}_{2}$.

Nowadays, the numerical modeling of photonics crystals is based on the calculation of the transmission, the reflection coefficients properties [19-23]. These methods including the plane wave expansion (PWE) method, the generalized Rayleigh identity method, the finite-difference time-domain (FDTD) method, and the transfer matrix method (TMM). Each method has its own limitations for finding the band structure. The transfer matrix method is most popular because of its simplicity in algorithm and capability to model complex structures. It is recently introduced by Pendry and MacKinnon, to calculate the EM transmission through the PBG materials [24].

In this paper, we combine the transfer matrix method (TMM) to the Block theorem in order to find the characteristics of transmission spectra and diagrams of dispersion of one dimensional photonic crystal (1D- PC). Several simulation cases by Matlab will be given to show the performance of this approach. The results obtained from this approach are in very good agreement with reported by the practical study of H. Tian et al. [14].

\section{Theory}

\subsection{Study of Transmissions in the (1D-PC) Structure}

Let us consider first the (1D-PC) structure consisting of alternating multilayer shown in Fig. 1, there are $\mathrm{N}$ layer or $(\mathrm{N} / 2)$ period made up of dielectric materials. Every layer has 
to be $d_{1}$ thicknesses, and index $n_{1}$. In order to find the formulation of the structure, we supposed that the incident electromagnetic wave from air to $\mathrm{Si}$ and $\mathrm{SiO}_{2}$ medium.

Let the layers be in the $\mathrm{x}-\mathrm{y}$ plane, the $\mathrm{z}$ direction being normal to interface of layers.

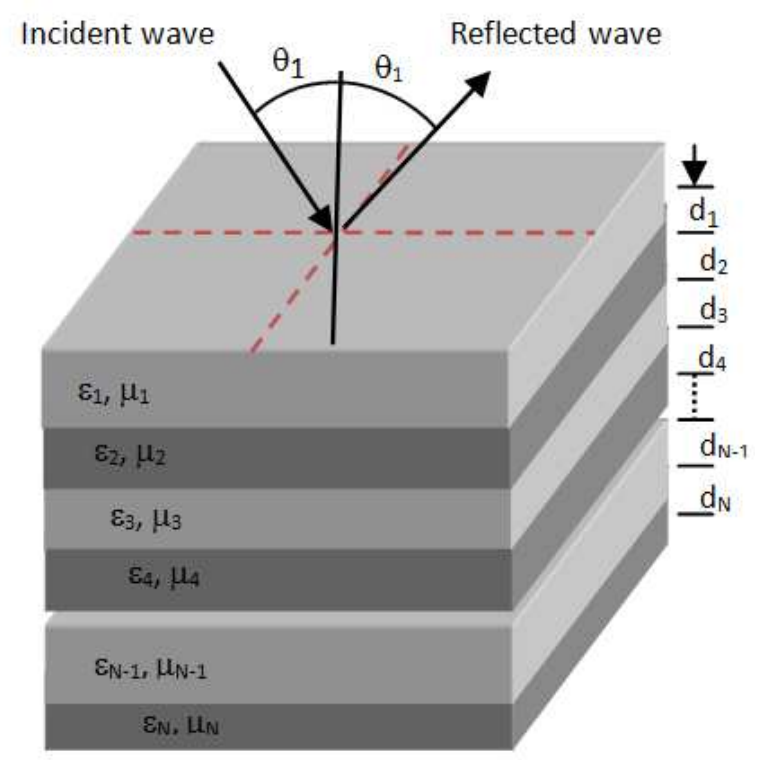

The refractive index profile of considered structure can be given as

$$
\varepsilon_{l}=\left\{\begin{array}{c}
\varepsilon_{1} 0<z<\mathrm{d}_{1} \\
\varepsilon_{2} \mathrm{~d}_{1}<z<\mathrm{d}_{2}
\end{array}\right.
$$

and

$$
\varepsilon_{l}(\mathrm{z})=\varepsilon_{l}(\mathrm{z}+d)
$$

where

1 : is number of layer

$\mathrm{d}=\mathrm{d}_{1}+\mathrm{d}_{2}$ : is period

Based on the Maxwell equations and the boundary conditions, the TMM has been widely used to calculate the amplitude and phase spectra of the light wave propagating in a (1D- PC) structure. We will suppose that a space time dependence of all the components of the kind $e^{i(\vec{k} \cdot \vec{r}-\omega t)}$. The transverse components of the $\mathrm{E}$ and $\mathrm{H}$ fields from Maxwell's equations in the lth layer, for TM polarization, are given by:

Fig. 1. Structure of one-dimensional photonic crystal.

$$
\begin{gathered}
\left.H_{l y}=A_{l} e^{i\left(\omega t-k_{l}\left(Z_{l} \cdot \cos \theta_{l}+X_{l} \cdot \sin \theta_{l}\right)\right)}+B_{l} e^{i\left(\omega t+k_{l}\left(Z_{l} \cdot \cos \theta_{l}+X_{l} \cdot \sin \theta_{l}\right)\right)}\right) \\
E_{l x}=\eta_{l} \cos \theta_{l}\left(A_{l} e^{i\left(\omega t-k_{l}\left(Z_{l} \cdot \cos \theta_{l}+X_{l} \cdot \sin \theta_{l}\right)\right)}-B_{l} e^{i\left(\omega t+k_{l}\left(Z_{l} \cdot \cos \theta_{l}+X_{l} \cdot \sin \theta_{l}\right)\right)}\right) \\
E_{l z}=-\eta_{l} \cos \theta_{l}\left(A_{l} e^{i\left(\omega t-k_{l}\left(Z_{l} \cdot \cos \theta_{l}+X_{l} \cdot \sin \theta_{l}\right)\right)}+B_{l} e^{i\left(\omega t+k_{l}\left(Z_{l} \cdot \cos \theta_{l}+X_{l} \cdot \sin \theta_{l}\right)\right)}\right)
\end{gathered}
$$

Where $A_{1}$ and $B_{1}$ are the amplitudes of the forward and backward travelling waves in the lth layer.

The transverse components of the $\mathrm{E}$ and $\mathrm{H}$ fields from

$$
\begin{gathered}
\left.E_{l y}=A_{l} e^{i\left(\omega t-k_{l}\left(Z_{l} \cdot \cos \theta_{l}+X_{l} \cdot \sin \theta_{l}\right)\right)}+B_{l} e^{i\left(\omega t+k_{l}\left(Z_{l} \cdot \cos \theta_{l}+X_{l} \cdot \sin \theta_{l}\right)\right)}\right) \\
H_{l x}=-\frac{\eta_{l}}{\cos \theta_{l}}\left(A_{l} e^{i\left(\omega t-k_{l}\left(Z_{l} \cdot \cos \theta_{l}+X_{l} \cdot \sin \theta_{l}\right)\right)}-B_{l} e^{i\left(\omega t+k_{l}\left(Z_{l} \cdot \cos \theta_{l}+X_{l} \cdot \sin \theta_{l}\right)\right)}\right) \\
H_{l z}=\frac{\eta_{l}}{\cos \theta_{l}}\left(A_{l} e^{i\left(\omega t-k_{l}\left(Z_{l} \cdot \cos \theta_{l}+X_{l} \cdot \sin \theta_{l}\right)\right)}+B_{l} e^{i\left(\omega t+k_{1}\left(Z_{l} \cdot \cos \theta_{l}+X_{l} \cdot \sin \theta_{l}\right)\right)}\right)
\end{gathered}
$$

Where the wave numbers and intrinsic impedances are:

$$
\begin{gathered}
k_{l}=\omega \sqrt{\varepsilon_{0} \mu_{0} \varepsilon_{1} \mu_{1}} \\
\eta_{1}=\frac{\mathrm{k}_{\mathrm{l}}}{\omega \varepsilon_{1} \varepsilon_{0}}=\sqrt{\frac{\mu_{0} \mu_{1}}{\varepsilon_{0} \varepsilon_{l}}}
\end{gathered}
$$

By using the boundary conditions and the condition of continuity of $\mathrm{E}$ and $\mathrm{H}$ fields at the interfaces of $\mathrm{z}=0$ and $\mathrm{z}=d_{1}, d_{2}, d_{3} \ldots \ldots . d_{N}$, we can found the relationship between the fields (1D- PC) structure consisting of 1 layer, this relation is given by:

$$
\left[\begin{array}{l}
E_{1} \\
H_{1}
\end{array}\right]=\mathrm{M}_{1} \mathrm{M}_{2} \ldots \ldots \mathrm{M}_{\mathrm{k}} \ldots \mathrm{M}_{\mathrm{l}-1} \mathrm{M}_{\mathrm{l}}\left[\begin{array}{l}
E_{l} \\
H_{l}
\end{array}\right]
$$

The matrix $\mathrm{M}_{1-1}$ of the $\mathrm{l}^{\text {th }}$ layer can be written in the form

$\delta_{(l-1)}$ and $\gamma_{(l-1)}$ being the matrix parameters and depending on the incident angle of light, the optical constants and the layer thickness, are expressed as:

$$
\begin{gathered}
\delta_{(l-1)}=k_{(l-1)} \cdot d_{(l-1)} \cdot \cos \theta_{(l-1)} \\
\gamma_{(l-1)}=\left\{\begin{array}{c}
\frac{\eta_{(l-1)}}{\cos \theta_{(l-1)}} \text { TE mode } \\
\eta_{(l-1)} \cos \theta_{(l-1)} \text { TM mode }
\end{array}\right.
\end{gathered}
$$

We note that $\theta_{(l-1)}$ is related to the angle of incidence $\theta_{0}$ by the Snell's Descart's low, that is 


$$
n_{(l-1)} \sin \theta_{(l-1)}=n_{0} \sin \theta_{0}
$$

By considering the transmission matrix of each layer, we can obtain the transmission matrix of whole structure. For 1 number of multilayers; the corresponding transfer matrix can be defined as a product of matrices, is obtained to be

$$
\prod_{k=1}^{l} M_{k}=\left[\begin{array}{ll}
\mathrm{m}_{11} & \mathrm{~m}_{12} \\
\mathrm{~m}_{21} & \mathrm{~m}_{22}
\end{array}\right]
$$

Where

$\mathrm{m}_{11}, \mathrm{~m}_{12}, \mathrm{~m}_{21}$ and $\mathrm{m}_{22}$ are the complex numbers

The transmittance $t$ and reflectance $r$ are defined as the ratios of the fluxes of the transmitted and reflected waves, respectively, to the flux of the incident wave. After some derivations, the total transmission and reflection coefficients are given by

$$
\begin{aligned}
& r=\frac{\left(m_{11}+p_{s}^{-1} m_{12}\right) p_{0}^{-1}-\left(m_{21}+p_{s}^{-1} m_{22}\right)}{\left(m_{11}+p_{s}^{-1} m_{12}\right) p_{0}^{-1}+\left(m_{21}+p_{s}^{-1} m_{22}\right)} \\
& t=\frac{2 \cdot p_{0}^{-1}}{\left(m_{11}+p_{s}^{-1} m_{12}\right) p_{0}^{-1}+\left(m_{21}+p_{s}^{-1} m_{22}\right)}
\end{aligned}
$$

Here $p_{0}$ and $p_{s}$ are the first and last medium of the structure which given as

$$
\begin{gathered}
p_{s}^{-1}=\left\{\begin{array}{l}
\frac{\eta_{s} \cos \theta_{s}}{Z_{0}} \text { TE mode } \\
\frac{\cos \theta_{s}}{\eta_{s} Z_{0}} \text { TM mode }
\end{array}\right. \\
p_{0}^{-1}=\left\{\begin{array}{l}
\frac{\eta_{0} \cos \theta_{0}}{Z_{0}} \text { TE mode } \\
\frac{\cos \theta_{0}}{\eta_{0} Z_{0}} \text { TM mode }
\end{array}\right.
\end{gathered}
$$

Where

$$
Z_{0}=\sqrt{\frac{\mu_{0}}{\varepsilon_{0}}}
$$

Hence the reflectance $\mathrm{R}$ and transmittance $\mathrm{T}$ spectrums of can be obtained by using the expressions:

$$
\begin{aligned}
& T=|t|^{2} \\
& R=|r|^{2}
\end{aligned}
$$

\subsection{The Dispersion Relation}

In general, wave propagation in periodic media can be described in terms of Bloch waves. For a determination of the dispersion surfaces of a periodic crystal, it is necessary only to integrate the wave-field through a periodic media [6].

According to Bloch theorem, fields in a periodic structure satisfy the following equations:

$$
E(z+d)=e^{-i k d} E(z)
$$

The parameter $k$ is called the Bloch wave number or dispersion relation. In order to determinate $k$, we can use relation between the electric field amplitudes of two layers. From Equation (11), we obtain:

$$
\left[\begin{array}{l}
E_{1} \\
H_{1}
\end{array}\right]=\mathrm{M}_{1} \mathrm{M}_{2}\left[\begin{array}{l}
E_{2} \\
H_{2}
\end{array}\right]
$$

We can put the product matrix as:

$$
M_{1} \cdot M_{2}=\left[\begin{array}{ll}
M_{11} & M_{12} \\
M_{21} & M_{22}
\end{array}\right]
$$

$\operatorname{Tr}\left[\mathrm{M}_{1} \cdot \mathrm{M}_{2}\right]$ is the trace of the transfer matrix characterizing the wave scattering in a periodic structure, is given by;

$$
\operatorname{Tr}\left[M_{1} \cdot M_{2}\right]=M_{11}+M_{22}=2 \cos (k d)
$$

Where

$$
\begin{gathered}
\mathrm{M}_{11}=\cos \left(\delta_{1}\right) * \cos \left(\delta_{2}\right)-\left(\gamma_{1} / \gamma_{2}\right) \sin \left(\delta_{1}\right) \sin \left(\delta_{2}\right) \\
\mathrm{M}_{22}=\cos \left(\delta_{1}\right) * \cos \left(\delta_{2}\right)-\left(\gamma_{2} / \gamma_{1}\right) \sin \left(\delta_{1}\right) \sin \left(\delta_{2}\right)
\end{gathered}
$$

Substituting (27) and (28) into (26), we obtain the following equation:

$\cos (\mathrm{kd})=\cos \left(\delta_{1}\right) * \cos \left(\delta_{2}\right)-\left(\frac{\gamma_{2}^{2}+\gamma_{1}^{2}}{2 \gamma_{1} \gamma_{2}}\right) \sin \left(\delta_{1}\right) \sin \left(\delta_{2}\right)$

The quantity $\cos (\mathrm{kd})$ determines the band structures of the (1D- PC) structure. In the region where $|\cos (\mathrm{kd})|<1, \mathrm{k}$ takes a real value and this leads to propagating Bloch waves (pass band). In the region where $|\cos (\mathrm{kd})|>1$, the value of $\mathrm{k}$ become complex which consists of an imaginary and a real part corresponding to the evanescent and propagating Bloch waves. The band edges are the regions where $|\cos (\mathrm{kd})|=1$.

\section{Numerical Calculation and Discussion}

\subsection{Transmission Properties Under Different Incident Angles}

In this subsection, we consider only normal incidence of the electromagnetic wave on the (1D- PC) structure. To check the correctness of our computer program, numerical results are compared with those obtained from the real values in the practical uses [14]. The structure is restructured as $\left(H_{n} L_{n}\right)^{m}$ where $\mathrm{n}=1 \ldots \mathrm{m}, \mathrm{m}$ is chosen as 15 . We have kept constant the dielectric permittivity's of the layers, are fixed to be $\mathrm{n}_{\mathrm{H}}=$ 3.7 and $\mathrm{n}_{\mathrm{L}}=1.5\left(\mathrm{Si}\right.$ and the second layer is $\mathrm{SiO}_{2}$ ). There are three different values of the thickness of the considered layers that we will use in our studies, are: $\left(\mathrm{d}_{\mathrm{H}}=112.1 \mathrm{~nm}, \mathrm{~d}_{\mathrm{L}}=276.4\right.$ $\mathrm{nm}),\left(\mathrm{d}_{\mathrm{H}}=112.1 \mathrm{~nm}, \mathrm{~d}_{\mathrm{L}}=281.4 \mathrm{~nm}\right)$ and $\left(\mathrm{d}_{\mathrm{H}}=117.1 \mathrm{~nm}, \mathrm{~d}_{\mathrm{L}}=\right.$ $281.4 \mathrm{~nm})$.

The transmission spectra in figures $2-4$ is computed and plotted with wavelength centered at $2.5 \mu \mathrm{m}$ taking into account the different values of the filling ratio $\left(F=d_{H} / d\right)$. It may be seen from these results, that the structure exhibits various band gaps (or stop band) where the photonic states are forbidden in the structure, can be seen in the transmission spectrum. In this study we are considered only the larger band gap width, is defined as the frequency range when $T \leq 0.01 \%$. When the thickness of the layer is $d_{H}=112.1 \mathrm{~nm}$, and $d_{L}=$ $276.4 \mathrm{~nm}$, the band gap rang is $1294 \mathrm{~nm}$ to $2321 \mathrm{~nm}$. When $\mathrm{d}_{\mathrm{H}}$ $=112.1 \mathrm{~nm}$ and $\mathrm{d}_{\mathrm{L}}=281.4 \mathrm{~nm}$, the band gap rang is $1394 \mathrm{~nm}$ to $2437 \mathrm{~nm}$. When $d_{H}=112.1 \mathrm{~nm}$ and $d_{L}=281.4 \mathrm{~nm}$, the band 
gap rang is $1463 \mathrm{~nm}$ to $2699 \mathrm{~nm}$. The photonic band gap is also same for both the TE and TM modes. So that, we can observe that the figure 4 has wider band gap than the figures 2-3. Also we can say that the change in the filling ratio allows us to obtain a new structure which can have adjustable gap width. It is found that the agreement between our results and those obtained via practical uses [14] is very good since the discrepancies between the two sets of results are below $0.8 \%$.

In this subsection, we consider only normal incidence of the electromagnetic wave on the (1D- PC) structure. To check the correctness of our computer program, numerical results are compared with those obtained from the real values in the practical uses [14]. The structure is restructured as $\left(H_{n} L_{n}\right)^{m}$ where $\mathrm{n}=1 \ldots \mathrm{m}, \mathrm{m}$ is chosen as 15 . We have kept constant the dielectric permittivity's of the layers, are fixed to be $n_{H}=$ 3.7 and $\mathrm{n}_{\mathrm{L}}=1.5\left(\mathrm{Si}\right.$ and the second layer is $\mathrm{SiO}_{2}$ ). There are three different values of the thickness of the considered layers that we will use in our studies, are: $\left(\mathrm{d}_{\mathrm{H}}=112.1 \mathrm{~nm}, \mathrm{~d}_{\mathrm{L}}=276.4\right.$ $\mathrm{nm}),\left(\mathrm{d}_{\mathrm{H}}=112.1 \mathrm{~nm}, \mathrm{~d}_{\mathrm{L}}=281.4 \mathrm{~nm}\right)$ and $\left(\mathrm{d}_{\mathrm{H}}=117.1 \mathrm{~nm}, \mathrm{~d}_{\mathrm{L}}=\right.$ $281.4 \mathrm{~nm})$.

The transmission spectra in figures 2-4 is computed and plotted with wavelength centered at $2.5 \mu \mathrm{m}$ taking into account the different values of the filling ratio $\left(\mathrm{F}=\mathrm{d}_{\mathrm{H}} / \mathrm{d}\right)$. It may be seen from these results, that the structure exhibits various band gaps (or stop band) where the photonic states are forbidden in the structure, can be seen in the transmission spectrum. In this study we are considered only the larger band gap width, is defined as the frequency range when $T \leq 0.01 \%$. When the thickness of the layer is $\mathrm{d}_{\mathrm{H}}=112.1 \mathrm{~nm}$, and $\mathrm{d}_{\mathrm{L}}=$ $276.4 \mathrm{~nm}$, the band gap rang is $1294 \mathrm{~nm}$ to $2321 \mathrm{~nm}$. When $\mathrm{d}_{\mathrm{H}}$ $=112.1 \mathrm{~nm}$ and $\mathrm{d}_{\mathrm{L}}=281.4 \mathrm{~nm}$, the band gap rang is $1394 \mathrm{~nm}$ to $2437 \mathrm{~nm}$. When $\mathrm{d}_{\mathrm{H}}=112.1 \mathrm{~nm}$ and $\mathrm{d}_{\mathrm{L}}=281.4 \mathrm{~nm}$, the band gap rang is $1463 \mathrm{~nm}$ to $2699 \mathrm{~nm}$. The photonic band gap is also same for both the TE and TM modes. So that, we can observe that the figure 4 has wider band gap than the figures 2-3. Also we can say that the change in the filling ratio allows us to obtain a new structure which can have adjustable gap width. It is found that the agreement between our results and those obtained via practical uses [14] is very good since the discrepancies between the two sets of results are below $0.8 \%$.

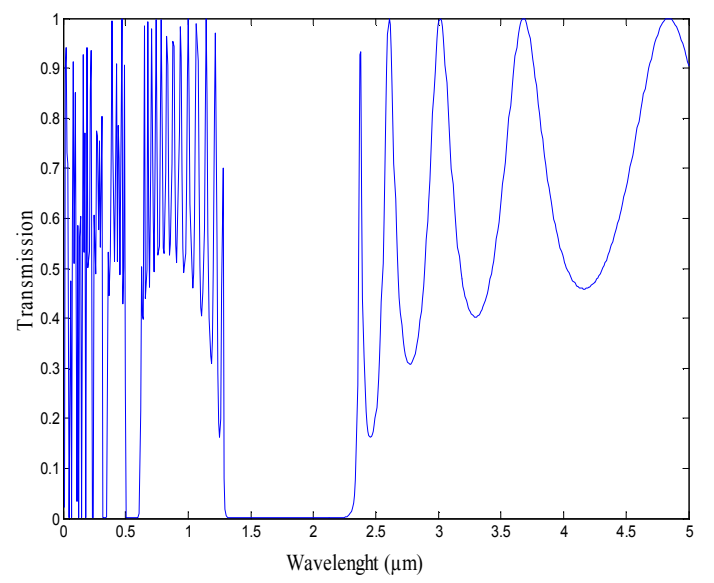

Fig. 2. Transmission spectra of $1 D, n_{H}=3.7, n_{L}=1.5, d_{H}=112.1 \mathrm{~nm}, d_{L}=$ $276.4 \mathrm{~nm}$ and $\mathrm{F}=0.288$.

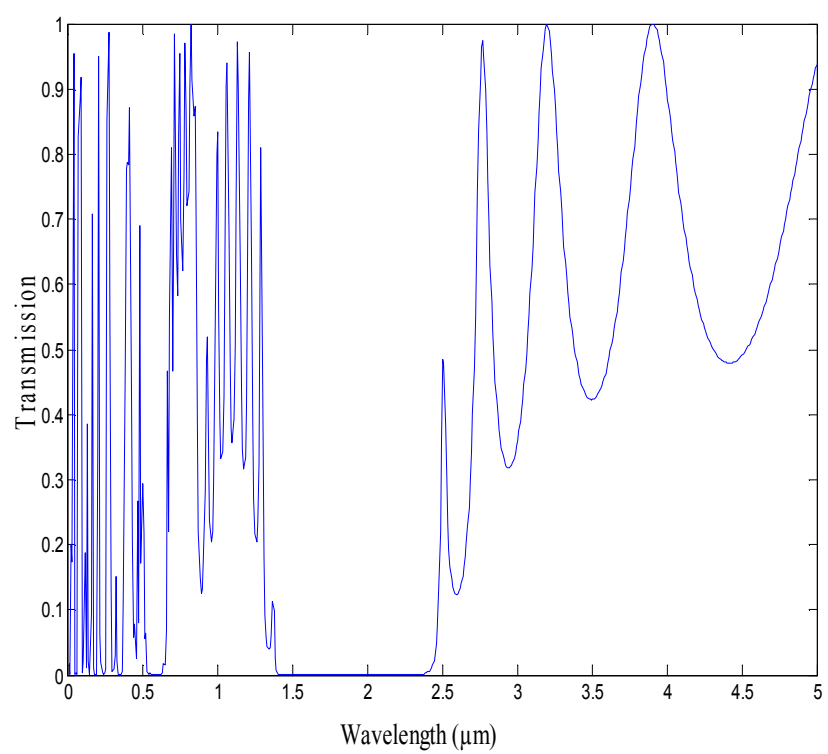

Fig. 3. Transmission spectra of $1 D, n_{H}=3.7, n_{L}=1.5, d_{H}=112.1 \mathrm{~nm}, d_{L}=$ $281.4 \mathrm{~nm}$, and $F=0.284$.

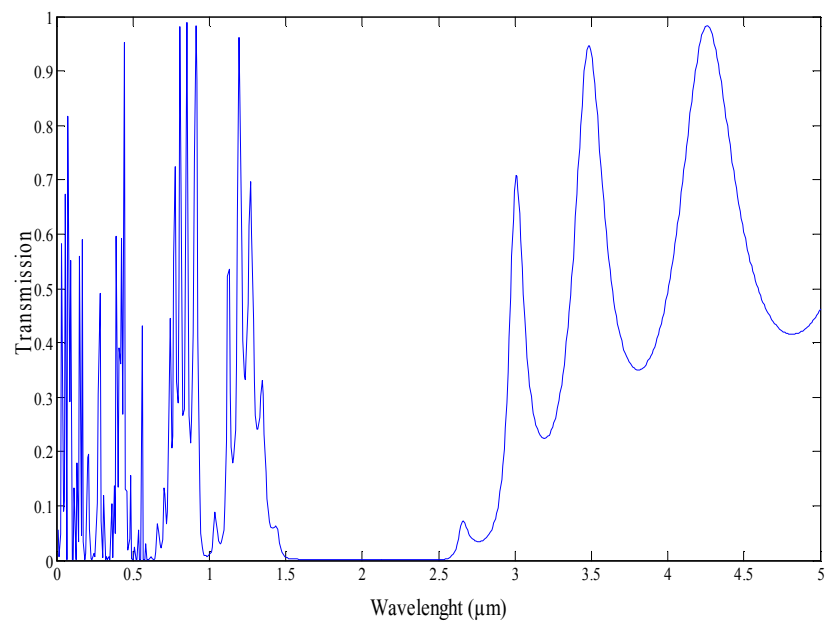

Fig. 4. Transmission spectra of $1 D, n_{H}=3.7, n_{L}=1.5, d_{H}=d_{H}=117.1 \mathrm{~nm}, d_{L}$ $=276.4 \mathrm{~nm}$, and $F=0.297$.

The angle of the incidence is an important factor in the enlargement of gap width that is omitted in the previous studies $[1,4,5,14]$. In the following, we present the results obtained of band gap width as functions of incident angle for two kinds (TE and TM modes). From the Figures 5-7, it is clearly seen that the band gap width for the TM mode is less than the TE mode for all the three cases. The band gap width of the structure compared for the incident angle with that in case of normal incidence is better, and the band gap width is more suitable when the angle is important for mode TE. The gap width of TM mode decreases with the increase of the angle for all the three cases. In addition, we can see that the band gap width of both modes (TE or TM) increases with the increase of filling ratio. 


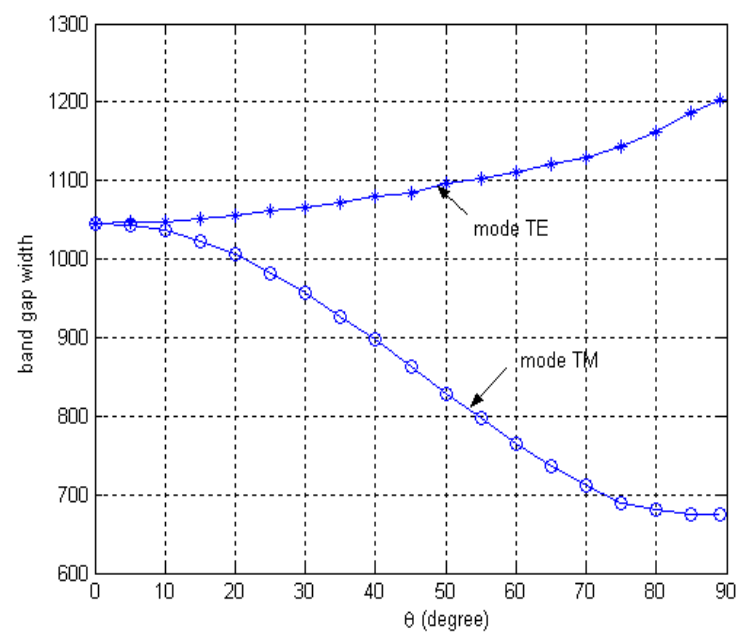

Fig. 5. Band gap width as functions of incident angle, $n_{H}=3.7, n_{L}=1.5, d_{H}=$ $112.1 \mathrm{~nm}$ and $d_{L}=276.4 \mathrm{~nm}$.

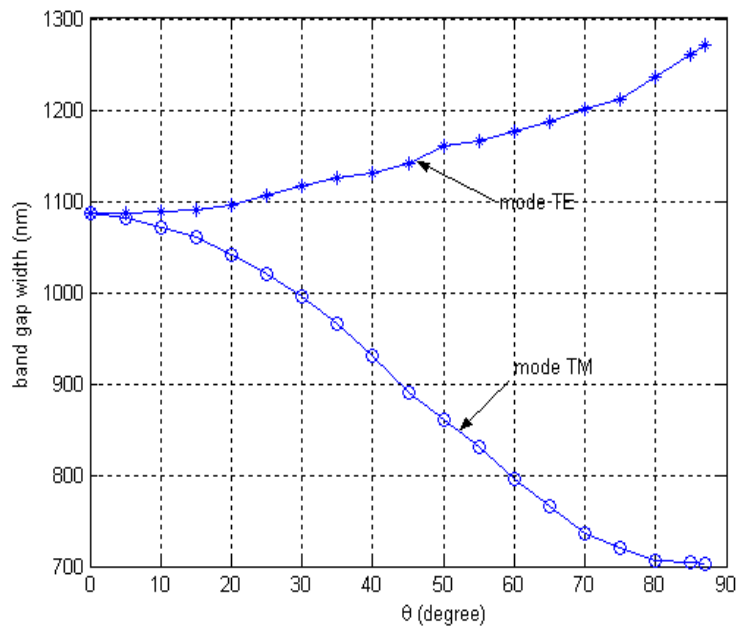

Fig. 6. Band gap width as functions of incident angle, $n_{H}=3.7, n_{L}=1.5, d_{H}=$ $112.1 \mathrm{~nm}$ and $d_{L}=281.4 \mathrm{~nm}$.

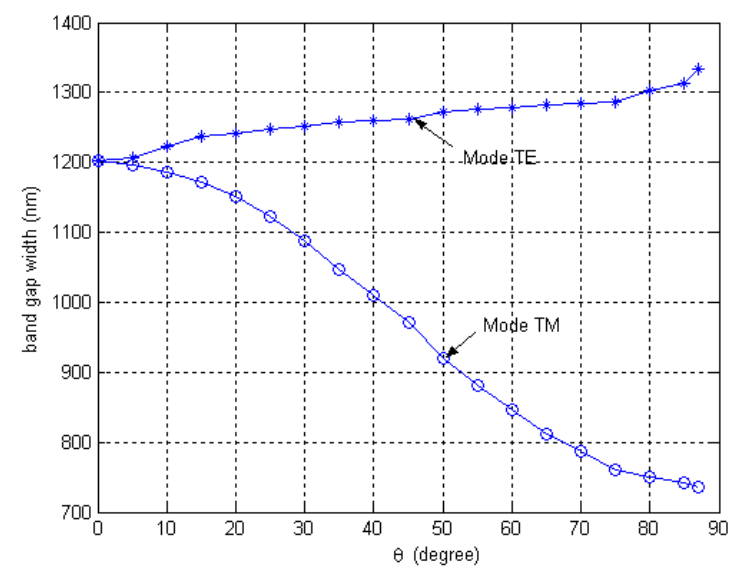

Fig. 7. Band gap width as functions of incident angle, $n_{H}=3.7, n_{L}=1.5, d_{H}=$ $117.1 \mathrm{~nm}$ and $d_{L}=276.4 \mathrm{~nm}$.

\subsection{Band Gap Structure of the (1D-PC) Structure}

To predict the propagation of an electromagnetic wave in a (1D- PC) structure, it is necessary to know its dispersion relation or $\omega(k)$, where $\omega$ is the frequency of a wave with wavenumber $k$. In order to determine the variation of dispersion diagram, we computed the normalized frequency versus the wave vector for transverse electric (TE) and transverse magnetic (TM) modes. It has been calculated for the first Brillouin zone by employing the transfer matrix method and Bloch's theorem.

In figures 8-10, results are presented for the dispersion diagram of the structure analyzed in fig 1 for normal incidence for TE (or TM) mode. The band diagram of figure 8 is extends between the normalized frequencies $(0.4344(\lambda / \mathrm{d})$ to 0.7713 $(\lambda / \mathrm{d}))$. In figure 9 , we have $(0.4294(\lambda / \mathrm{d})$ to $0.7631(\lambda / \mathrm{d}))$. In figure 10 , we have $(0.4245(\lambda / \mathrm{d})$ to $0.7532(\lambda / \mathrm{d}))$.

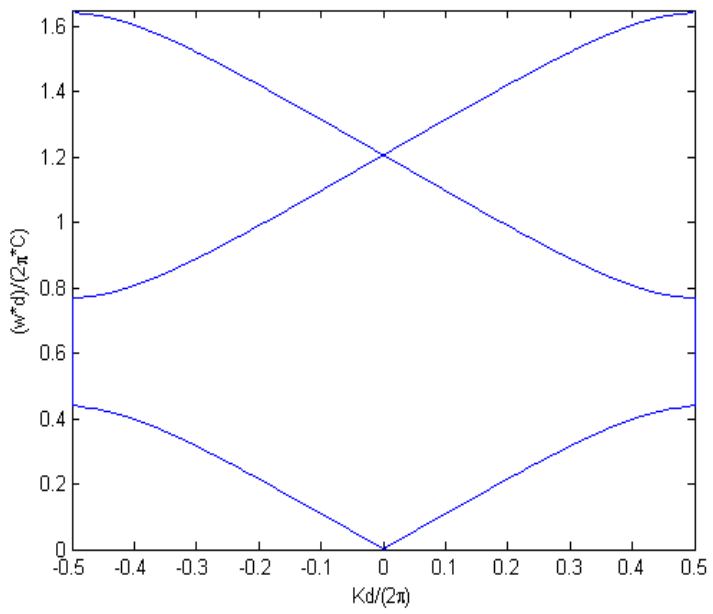

Fig. 8. Photonic band structure of (1D- PC), $n_{H}=3.7, n_{L}=1.5, d_{H}=112.1 \mathrm{~nm}$ and $d_{L}=276.4 \mathrm{~nm}, \theta=0^{\circ}$.

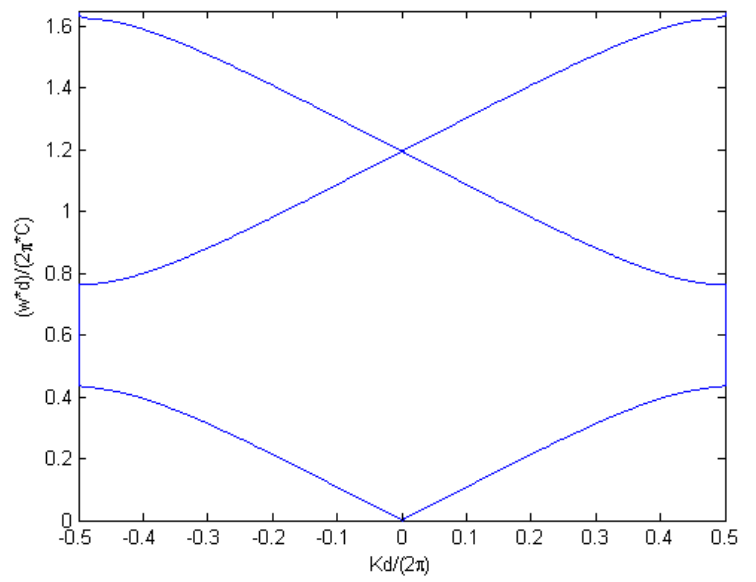

Fig. 9. Photonic band structure of $(1 D-P C), n_{H}=3.7, n_{L}=1.5, d_{H}=117.1 \mathrm{~nm}$ and $d_{L}=281.4 \mathrm{~nm}, \theta=0^{\circ}$.

As seen in these figures, a gap is present between the allowed states which can be easily identified, this corresponds to the band gap of the structure, and comparison shows that these gaps occur over the same frequency range as the transmission stop band. The existence of photonic band gap appeared when the value of Bloch wave vector becomes complex. However, the real values of Bloch wavevector $\mathrm{k}$ are corresponding to the pass band and imaginary values are corresponding to the forbidden band gap. 


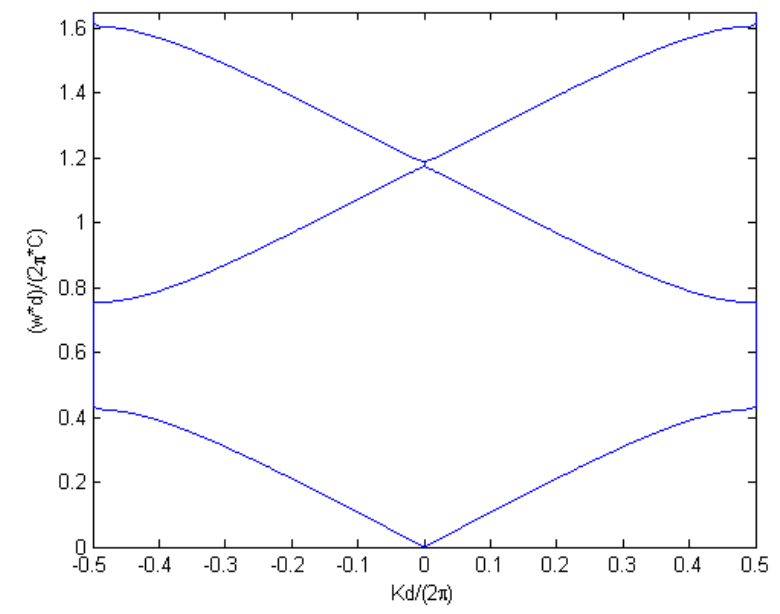

Fig. 10. Photonic band structure of $(1 D-P C)(1 D-P C), n_{H}=3.7, n_{L}=1.5, d_{H}$ $=117.1 \mathrm{~nm}$ and $d_{L}=276.4 \mathrm{~nm}, \theta=0^{\circ}$.

\subsection{Photonic Gap Map of the (1D-PC) Structure}

In this section, we have demonstrated that it is possible to modify the photonic gap (PBG) of structure by varying the incident angle and filling factor $f$. The gap map shown in Figures 11-13 represent results in terms of frequencies $\left(\frac{f \mathrm{~d}}{\mathrm{C}}\right)$ (where $d$ is the period constant, $\mathrm{C}$ the speed of light and $f$ the frequency) as function of the filling factor $\left(f=\mathrm{d}_{\mathrm{H}} / \mathrm{d}\right)$. The photonic gap map for the TE or TM mode (normal incidence), presented in figure 11, the red region indicates the variation of band gap (PBG), the empty space regions represent the ranges of transmission. From simulation analysis, we observe that the increasing of $f$ has the effect of increasing photonic gap. Also, we found that the first band gap is appear when the value of $f$ is greater than 0.001 .

In the figures 12-13, the red region indicates the variation of TE PBG, and the blue region indicates the variation of TM PBG for oblique incidence $\left(\theta=20^{\circ}\right)$. It may be seen from the plot that the forbidden bandwidth varies with the ratio filling factor $\left(d_{H} / d\right)$ of the two modes. As the angle of the incidence increases the forbidden bandwidth was also found to be increased and shifted to higher frequency regions

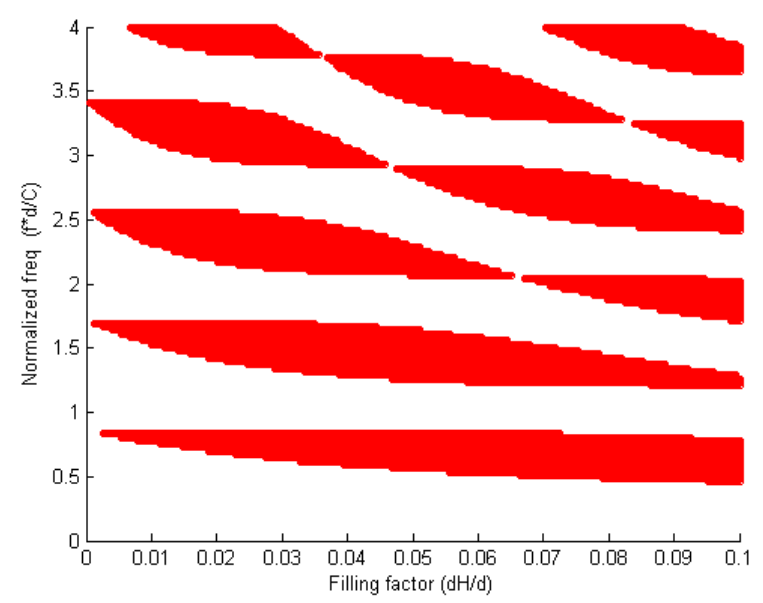

Fig. 11. Photonic gap map (normalized frequency $w a / 2 \pi c$ versus the filling factor $\left(d_{H} / d\right)$ of $(1 D-P C), n_{H}=3.7, n_{L}=1.5, d=388.5 \mathrm{~nm}, \theta=0^{\circ}$.

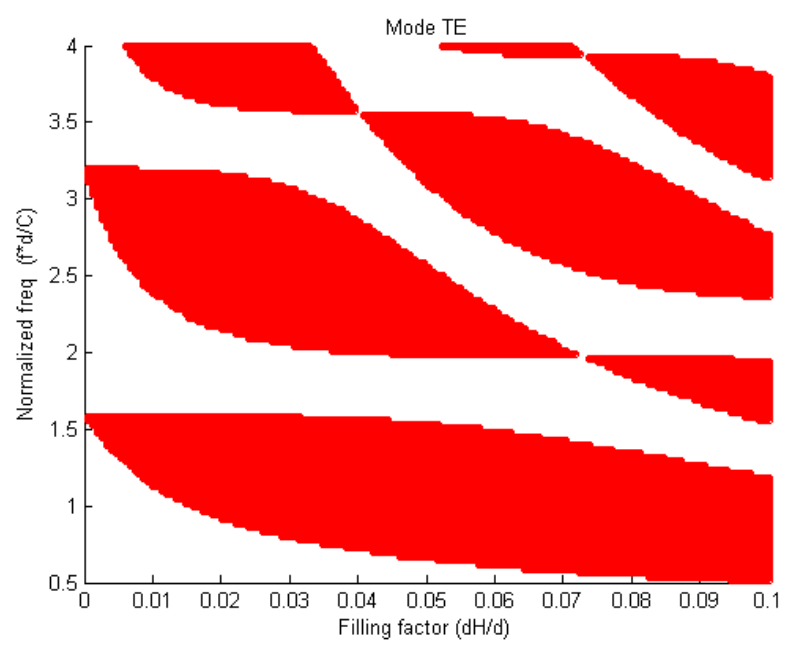

Fig. 12. Photonic gap map (normalized frequency $w a / 2 \pi c$ versus the filling factor $\left(d_{H} / d\right)$ of $(1 D-P C), n_{H}=3.7, n_{L}=1.5, d=388.5 \mathrm{~nm}$, Mode TE, $\theta=20^{\circ}$.

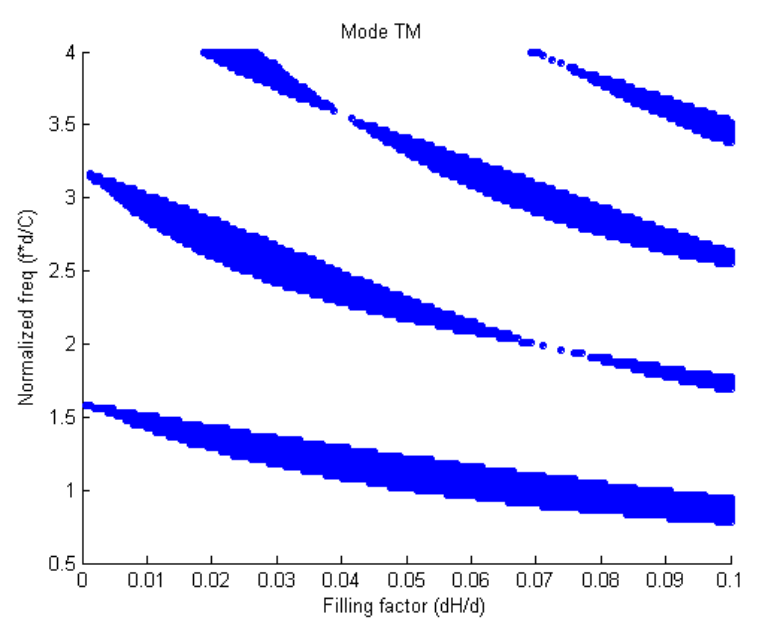

Fig. 13. Photonic gap map (normalized frequency $w a / 2 \pi c$ versus the filling factor $\left(d_{H} / d\right)$ of $(1 D-P C), n_{H}=3.7, n_{L}=1.5, d=388.5 \mathrm{~nm}$, Mode TM, $\theta=20^{\circ}$.

\section{Conclusion}

In summary, we have used the transfer matrix method and Bloch theorem to study one-dimensional photonic crystals. We have discussed the calculation of transmissivity, reflectivity, and dispersion relation for both TE and TM modes at different incidence angles. Our results show that the PBG sensitivity depend on many factors, such as the filling factor and polarization modes. The angle of incidence of the wave is also another factor which affects the width of band gaps. Further, theory approach TMM and Bloch theorem can allow for better characterization of the one-dimensional photonic crystals with defect.

\section{References}

[1] H. Oraizi A. Abdolali, "Several theorems for reflection and transmission coefficients of plane wave incidence on planar multilayer metamaterial structures", IET Microw. Antennas Propag., Vol. 4, Iss. 11, pp. 1870-1879, 2010. 
[2] I. V. Shadrivov, A. A. Sukhorukov, and Y. S. Kivshar, "Complete band gaps in one-dimensional left-handed periodic structures," Physical Review Letters, Vol. 95, pp. 1-4, 2005.

[3] Z. Wang, D. Liu "A few points on omnidirectional band gaps in one- dimensional photonic crystals," Applied Physics B, Appl. Phys. B 86, pp 473-476, 2007.

[4] A. Gharaati and Z. Zare, "Photonic band structures and enhancement of omnidirectional reflection bands by using a ternary 1D photonic crystal including left-handed materials", Progress In Electromagnetics Research M, Vol. 20, pp 81-94, 2011.

[5] S. K. Srivastava, and S. P. Ojha, "Enhancement of omnidirectional reflection bands in one-dimensional photonic crystals with left-handed materials," Progress In Electromagnetics Research, Vol. 68, pp 91-111, 2007.

[6] Sakoda K: Optical properties of Photonic Crystals. New York: Springer Berlin Heidlberg; 2005.

[7] M. Skorobogatiy, and J. Yang, Fundamentals of Photonic Crystal Guiding, Cambridge University Press, New York, 2009.

[8] I. A. Sukhovanov, and I. V. Guryev, Photonic Crystals Physics and Practical Modeling, Springer Series in Optical Science, New York, 2009.

[9] R. Gonalo, P. D. Maagt, M. Sorrolo, "Enhanced Patch antenna perfomnce by suppressing surface waves using PBG substrates," IEEE transactions on microwave theory and techniques. Vol. 47, No. 11, pp. 2131-2138, November 1999.

[10] E. Yablonovitch, T.J. Gmitter, "Photonic band structure: The face centered cubic case employing nonspherical atoms," J. Physical review letters, Vol.67, $\mathrm{N}^{\circ} 17$, pp. 2295-2298, 1991.

[11] K. M. Ho, C. T. Chan, and C. M. Soukoulis, "Existence of a Photonic Gap in Periodic Dielectric Structures,"Phys Rev Lett, 1990 Dec 17, vol 65, N²5, pp 3152-3155.

[12] Costas M. Soukoulis, "Photonic Band Gap Materials," Proceedings of the NATO Advanced Study Institute on Photonic Band Gap Materials, Elounda, Crete, Greece, June 18-30, 1995.

[13] C. v. Mee, P. Contu, P. Pintus, "One-dimensional photonic crystal design", Journal of Quantitative Spectroscopy \& Radiative Transfer, Vol. 111, pp. 214-225, 2010.
[14] H. Tian, Y. Ji, C. Li, H. Liu, "Transmission properties of one-dimensional graded photonic crystals and enlargement of omnidirectional negligible transmission gap", Elsevier, Optics Communications Vol 275, N¹, pp 83-89, July 2007.

[15] B. Gallas S. Fission, E. Charron, A. Brunet-Bruneau, R. Vuye and J. Revory, " Making an omnidirectional reflector", Appl. Opt. 40, 5056-5063, 2001.

[16] K. M.Chen, A. W. Sparks, H. C. Luan, D. R. Lim, K. wada, and L. C. Kimerling, "SiO2/TiO2 omni-directional reflector and microcavity resonator via the sol-gel method," Appl. Phys. Lett., Vol. 75, pp. 3805-3807, 1999.

[17] D. N. Chigrin, A. V. Lavrinenko, D. A. Yarotsky, and S. V. Gaponenko, "Observation of total omni-directional reflection from a one-dimensional dielectric lattice," Appl. Phys. A:Mater. Sci. Process., Vol. 68, pp. 25-28, 1999.

[18] D. N. Chigrin, A. V. Lavrinenko, D. A. Yarotsky, and S. V. Gaponenko, "All dielectric one dimensional periodic structures for total omnidirectional reflection and partial spontaneous emission control, " J. Lightwave Tech., Vol. 17, pp.2018-2024, 1999.

[19] F. Scotognella, "Four-material one dimensional photonic crystals," Elsevier, Optical Materials, Vol. 34, N 9, July 2012, pp 1610-1613.

[20] K. M. Leung, and Y. F. Liu, "Photon band structures: The plane wave method", Physical Review B, 41, pp. 10188-10190, 1990.

[21] S. Şimşek, "A novel method for designing one dimensional photonic crystals with given bandgap characteristics," Elsevier, Int. J. Electron. Commun. (AEÜ), Vol. 67, pp827- 832, 2013.

[22] L. C. Botten, T. P. White, A. A. Asatryan, T. N. Langtry, C. M. de Sterke and R. C. McPhedran, "Bloch mode scattering matrix methods for modeling extended photonic crystal structures. Part I: Theory," Phys. Rev. E, Vol. 70, 056606, 2004.

[23] L. C. Botten, N. A. Nicorovici, R. C. McPhedran, A. A. Asatryan, and C. M. de Sterke, "Photonic band structure calculations using scattering matrices", Phys. Rev. E, Vol. 64, 046603, pp. 1-20, 2001.

[24] J. B. Pendry, A. Mackinnon, "Calculation of photon dispersion, "Phys. Rev. Lett., 69, (3), pp. 2772-2775, 1992. 